\title{
CONVERGENCE OF PRODUCT INTEGRATION RULES OVER $(0, \infty)$ FOR FUNCTIONS WITH WEAK SINGULARITIES AT THE ORIGIN
}

\author{
G. MASTROIANNI AND G. MONEGATO
}

Dedicated to Luigi Gatteschi on his 70th birthday

ABSTRACT. In this paper we consider integrals of the form

$$
\int_{0}^{\infty} e^{-x} K(x, y) f(x) d x
$$

with $f \in C^{p}[0, \infty) \cap C^{q}(0, \infty), q \geq p \geq 0$, and $x^{i} f^{(p+i)}(x) \in C[0, \infty)$, $i=1, \ldots, q-p$, when $q>p$. They appear for instance in certain WienerHopf integral equations and are of interest if one wants to solve these by a Nyström method.

To discretize the integral above, we propose to use a product rule of interpolatory type based on the zeros of Laguerre polynomials. For this rule we derive (weighted) uniform convergence estimates and present some numerical examples.

\section{INTRODUCTION}

Several problems of mathematical physics lead to integral equations of the form

$$
u(y)-\int_{a}^{b} K(x-y) u(x) d x=h(y),
$$

where the kernel $K(x-y)$ is weakly singular at $x=y$, and the interval $(a, b)$ is either bounded or unbounded.

When $(a, b)$ is finite, for example $(a, b) \equiv(-1,1)$, and the input function $h(y)$ is smooth, say $h \in C^{m}[-1,1], m \geq 1$, the solution $u(x)$ of $(1.1)$ has the same degree of smoothness as $h(y)$ in $(-1,1)$, but exhibits some mild singularities at the endpoints \pm 1 . For instance, when $|K(x-y)| \leq c|x-y|^{\nu}$ for some $-1<\nu<0$, it has been shown in [27] that the solution $u(x)$ belongs to the set $C[-1,1] \cap C^{m}(-1,1)$. By taking advantage of known information on the behavior of $u(x)$ in $[-1,1]$, we have in [19] considered product integration rules of interpolatory type, based on the zeros of Jacobi polynomials, and have derived corresponding uniform convergence estimates. In the more general case of a function $u \in C^{p}[-1,1] \cap C^{q}(-1,1), p \geq 0, q \geq 2 p+2$, with $\left(1-x^{2}\right)^{i} u^{(p+i)}(x) \in C[-1,1], i=1, \ldots, q-p$, and a kernel $|K(x, y)| \leq$

Received by the editor December 2, 1993 and, in revised form, January 26, 1994.

1991 Mathematics Subject Classification. Primary 65D32, 65R20.

Work performed under the auspices of the Ministero dell'Università e della Ricerca Scientifica e Tecnologica of Italy. 
$c|x-y|^{\nu},-1<\nu<0$, we have obtained for the remainder term of the quadrature rule the following estimates:

$$
\begin{cases}O\left(n^{-2-2 p-2 \nu} \log n\right) E_{n-q}\left(\Phi^{(q)}\right) & \text { for }-1 \leq y \leq 1, \\ O\left(n^{-2-2 p} \log n\right) E_{n-q}\left(\Phi^{(q)}\right) & \text { for }-1+\varepsilon \leq y \leq 1-\varepsilon,\end{cases}
$$

where $E_{k}(f)$ denotes the error of the uniform best approximation polynomial of degree $k$ associated with the function $f$, and $\Phi(x)=\left(1-x^{2}\right)^{q-p} u(x)$. We recall that when $\Phi^{(q)}(x)$ is Hölder continuous of order $\mu$ in $[-1,1]$, we have $E_{n-q}\left(\Phi^{(q)}\right)=O\left(n^{-\mu}\right)$ (see [25]). Estimates like (1.2) are of importance when the product rule mentioned above is used to construct a stable Nyström interpolant to solve (1.1).

In the case of $(a, b)$ unbounded, a few authors (see [16, 23]) have considered product integration formulas based on zeros of orthogonal polynomials and proved their convergence. However, they have not derived any error estimates; furthermore, their studies essentially refer to the case $(a, b) \equiv(-\infty, \infty)$.

In this paper we consider the case of an integral of the form

$$
\int_{0}^{\infty} e^{-x} K(x, y) f(x) d x, \quad 0 \leq y<\infty,
$$

with

$$
\left\|e^{-\alpha y} \int_{0}^{\infty} e^{-x}|K(x, y)|^{2} d x\right\|_{\infty} \leq c \text { for some } \alpha \geq 0,
$$

associated with an integral equation of type (1.1). Indeed, in [13] it has been shown that when in (1.1) $K(x-y)$ satisfies some specified conditions, and the input function $h(y)$ is smooth on $(0, \infty)$, behaving like $c+y^{\mu}, 0<\mu<1$, as $y \rightarrow 0$, and like $e^{-\delta y}, \delta>0$, as $y \rightarrow \infty$, then the solution $u(x)$ has a similar behavior. If $h(y)$ is constant, then $\bar{u}(x)=u(x)-u(\infty)$ has the behavior described in the previous case, and the integral equation can be reformulated in the new unknown $\bar{u}(x)$.

Therefore, in $\S 2$ we examine the general case of $(1.3)$ with $f \in C^{p}[0, \infty) \cap$ $C^{q}(0, \infty), q \geq p \geq 0$, and $x^{i} f^{(p+i)}(x) \in C[0, \infty), i=1, \ldots, q-p$, and propose to approximate (1.3) by a product quadrature formula of interpolatory type based on the zeros of Laguerre polynomials. This rule will integrate exactly the factor $e^{-x} K(x, y)$. For its remainder term we obtain error estimates analogous to (1.2). In $\S 3$ we present some numerical examples which show how our product rules can be constructed and how they perform.

To derive the error estimates for our product rule, we use two intermediate results, which appear to be new and of interest in their own right: a weighted (with weight $e^{-x}$ ) $L^{2}$-convergence estimate for the Lagrange interpolation polynomial based on the zeros of Laguerre polynomials, and an estimate for the remainder term of the classical Gauss-Laguerre quadrature formula.

\section{Convergence estimates}

The following two lemmas, which are fundamental for deriving the convergence estimates in this section, have been very recently proved in [5].

Lemma 1. Let $g \in C^{q}[0, \infty)$. Then for all integers $m$ sufficiently large there exist polynomials $Q_{m}(x)$ of degree $m$ such that for $k=0, \ldots, q, r>0$, and 
$0 \leq x<\infty$,

$$
e^{-\frac{x}{r}} x^{\frac{k-q}{2}}\left|g^{(k)}(x)-Q_{m}^{(k)}(x)\right| \leq M_{q, k} m^{\frac{k-q}{2}} E_{m-q}\left(g^{(q)} ; e^{-\frac{x}{r}}\right),
$$

where $M_{q, k}$ is independent of $m$ and $g$, and ${ }^{1}$

$$
E_{n}(f ; w):=\inf _{p_{n}}\left\|w\left[f-p_{n}\right]\right\|_{\infty,[0, \infty)}
$$

Moreover, $g^{(q)}(0)=Q_{m}^{(q)}(0)$.

This result represents the half-line analogue of corresponding estimates derived, for example, in [12,7] in the case of finite intervals. As in [19], we will use bound (2.1) to obtain our convergence estimates.

Lemma 2. Let $\bar{g} \in C^{s}(-\infty, \infty)$. Then for all integers $m$ sufficiently large there exist polynomials $p_{m}(x)$ of degree $m$ such that for $k=0, \ldots, s, r>0$, and $-\infty<t<\infty$, we have

$$
\left|e^{-\frac{t^{2}}{r}}\left[\bar{g}^{(k)}(t)-p_{m}^{(k)}(t)\right]\right| \leq \gamma_{k} m^{\frac{k-s}{2}} E_{m-s}^{*}\left(\bar{g}^{(s)} ; e^{-\frac{t^{2}}{r}}\right)
$$

and

$$
\bar{g}^{(k)}(0)=p_{m}^{(k)}(0)
$$

where $\gamma_{k}$ is a constant independent of $\bar{g}$ and $m$, and

$$
E_{n}^{*}(f ; w):=\inf _{p_{n}}\left\|w\left[f-p_{n}\right]\right\|_{\infty,(-\infty, \infty)} .
$$

Furthermore, $p_{m}(t)$ can be chosen to be even if $\bar{g}(t)$ is even, and odd if $\bar{g}(t)$ is odd.

Lemma 2 is of importance because it allows us to establish a relationship between $E_{n}(g(x) ; w(x))$ and $E_{2 n}^{*}\left(g\left(t^{2}\right) ; w\left(t^{2}\right)\right)$. We recall that in the literature we find mainly estimates for this latter error (see for instance [9]). In our case, by setting $x=t^{2}$ and applying Lemma 2 with $s=k=0$ and $m=2 n$, we have $^{2}$

$$
E_{n}\left(g(x) ; e^{-\frac{x}{r}}\right) \leq c E_{2 n}^{*}\left(\bar{g}(t) ; e^{-\frac{t^{2}}{r}}\right),
$$

where we have defined $\bar{g}(t)=g\left(t^{2}\right)$. Bounds for $E_{n}^{*}$ can be obtained by using the representations given in [9, Chapter 11]. For instance, we have $E_{n}^{*}\left(t^{\mu} ; e^{-\frac{t^{2}}{r}}\right)=O\left(n^{-\frac{\mu}{2}}\right), 0<\mu<1$, and $E_{n}^{*}\left(t \log t ; e^{-\frac{t^{2}}{r}}\right)=O\left(n^{-\frac{1}{2}}\right)$.

Next, for $q \geq p \geq 0$ we set

$$
\begin{array}{r}
C_{p}^{q}[0, \infty):=\left\{f \in C^{p}[0, \infty) \cap C^{q}(0, \infty): x^{i} f^{(p+i)}(x) \in C[0, \infty),\right. \\
i=1, \ldots, q-p\},
\end{array}
$$

and with $f \in C_{p}^{q}[0, \infty)$ we associate the auxiliary function

$$
\Phi(x):=x^{q-p} f(x) \in C^{q}[0, \infty),
$$

which satisfies the conditions

$$
\Phi^{(i)}(0)=0, \quad i=0, \ldots, q-p-1,
$$

\footnotetext{
${ }^{1}$ Here, $p_{n}$ denotes a polynomial of degree $n$ and $\|u\|_{\infty,[0, \infty)}=\max _{0 \leq x<\infty}|u(x)|$.

${ }^{2}$ Here and in the following, $c$ denotes a constant taking different values on different occurrences.
} 
when $q>p$. We denote by $Q_{m}(x)$ the polynomial of degree $m$ defined by Lemma 1 with $g(x) \equiv \Phi(x)$. We have

$$
Q_{m}(x)=x^{q-p} P_{m+p-q}(x),
$$

where $P_{m+p-q}(x)$ is a polynomial of degree $m+p-q$. From (2.1) we obtain

$$
e^{-\frac{x}{r}}\left|\Phi^{(k)}(x)-Q_{m}^{(k)}(x)\right| \leq M_{q, k} x^{\frac{q-k}{2}} m^{\frac{k-q}{2}} E_{m-q}\left(\Phi^{(q)} ; e^{-\frac{x}{r}}\right) ;
$$

hence, recalling (2.4) and (2.5), and setting $k=0$ in (2.6), we have

$$
e^{-\frac{x}{r}}\left|f(x)-P_{m+p-q}(x)\right| \leq M_{q, 0} x^{p-\frac{q}{2}} m^{-\frac{q}{2}} E_{m-q}\left(\Phi^{(q)} ; e^{-\frac{x}{r}}\right) .
$$

Then we introduce the Lagrange interpolation polynomial of degree $n-1$, $L_{n}(f ; x)$, associated with the zeros of the Laguerre polynomial $L_{n}(x)$. We recall that the $L^{2}$-convergence, with weight $e^{-x}$, of $L_{n}(f ; x)$ towards $f(x)$ follows from a result derived many years ago in [3]. Indeed, if in the theorem of that paper we take $h(x) \equiv 0$ for $-\infty<x<0$ and $h(x)=e^{-\varepsilon x}, 0<\varepsilon<1$, for $0 \leq x<\infty$, and $g(x)=e^{(1-\varepsilon)|x|}$, we obtain

Lemma 3. Let $f \in C[0, \infty)$ be a function satisfying the inequality

$$
|f(x)| \leq c e^{(1-\varepsilon) \frac{x}{2}}, \quad 0 \leq x<\infty,
$$

with $\varepsilon>0$ as small as we like. Then we have

$$
\lim _{n \rightarrow \infty} \int_{0}^{\infty} e^{-x}\left[f(x)-L_{n}(f ; x)\right]^{2} d x=o(1) .
$$

Here, having inequality (2.7), we will derive the following estimate.

Theorem 1. Let $f \in C_{p}^{q}[0, \infty), q \geq p \geq 0$. Then for any $r>2$ we have, with $\Phi$ as in (2.4),

$$
\begin{aligned}
& \left\{\int_{0}^{\infty} e^{-x}\left[f(x)-L_{n}(f ; x)\right]^{2} d x\right\}^{\frac{1}{2}} \\
& \quad= \begin{cases}O\left(n^{-\frac{q}{2}}\right) E_{n-p-1}\left(\Phi^{(q)} ; e^{-\frac{x}{r}}\right) & \text { if } q \leq 2 p, \\
O\left(n^{-p-\frac{1}{2}} \log n\right) E_{n-p-1}\left(\Phi^{(q)} ; e^{-\frac{x}{r}}\right) & \text { if } q=2 p+1, \\
O\left(n^{-p-\frac{1}{2}}\right) E_{n-p-1}\left(\Phi^{(q)} ; e^{-\frac{x}{r}}\right) & \text { if } q \geq 2 p+2 .\end{cases}
\end{aligned}
$$

Proof. Let $P_{n-1}(x)$ be the polynomial of degree $n-1$ defined in (2.5), and write

$$
\begin{aligned}
& \left\{\int_{0}^{\infty} e^{-x}\left[f(x)-L_{n}(f ; x)\right]^{2} d x\right\}^{\frac{1}{2}} \\
& \quad \leq\left\{\int_{0}^{\infty} e^{-x}\left[f(x)-P_{n-1}(x)\right]^{2} d x\right\}^{\frac{1}{2}}+\left\{\int_{0}^{\infty} e^{-x}\left[L_{n}\left(f-P_{n-1} ; x\right)\right]^{2} d x\right\}^{\frac{1}{2}} .
\end{aligned}
$$

Thus we need to examine the behavior of the following two integrals:

$$
\begin{aligned}
& I_{1}=\int_{0}^{\infty} e^{-x}\left[f(x)-P_{n-1}(x)\right]^{2} d x, \\
& I_{2}=\int_{0}^{\infty} e^{-x}\left[L_{n}\left(f-P_{n-1} ; x\right)\right]^{2} d x .
\end{aligned}
$$


We consider first $I_{1}$ and break the interval of integration in three parts: $\left[0, n^{-1}\right]$, $\left[n^{-1}, 1\right],[1, \infty)$. Then we proceed as follows. For $0 \leq x \leq n^{-1}$ we write

$$
\begin{aligned}
e^{-\frac{x}{r}}\left|f(x)-P_{n-1}(x)\right| & =e^{-\frac{x}{r}} \frac{\left|\Phi(x)-Q_{n+q-p-1}(x)\right|}{x^{q-p}} \\
& =e^{-\frac{x}{r}} \frac{\left|\Phi^{(q-p)}\left(\xi_{x}\right)-Q_{n+q-p-1}^{(q-p)}\left(\xi_{x}\right)\right|}{(q-p) !} ;
\end{aligned}
$$

hence, recalling (2.6) with $k=q-p$, we get

$$
e^{-\frac{x}{r}}\left|f(x)-P_{n-1}(x)\right| \leq c x^{\frac{p}{2}} n^{-\frac{p}{2}} E_{n-p-1}\left(\Phi^{(q)} ; e^{-\frac{x}{r}}\right) .
$$

From this inequality we derive the bound

$$
\int_{0}^{n^{-1}} e^{-x}\left[f(x)-P_{n-1}(x)\right]^{2} d x \leq c n^{-2 p-1} E_{n-p-1}^{2}\left(\Phi^{(q)} ; e^{-\frac{x}{r}}\right), \quad r \geq 2 .
$$

In the case $n^{-1} \leq x \leq 1$ we use (2.7) and obtain first, for $r \geq 2$,

$$
\int_{n^{-1}}^{1} e^{-x}\left[f(x)-P_{n-1}(x)\right]^{2} d x \leq c\left[\int_{n^{-1}}^{1} x^{2 p-q} d x\right] n^{-q} E_{n-p-1}^{2}\left(\Phi^{(q)} ; e^{-\frac{x}{r}}\right),
$$

and then the bounds

$$
\begin{cases}O\left(n^{-q}\right) E_{n-p-1}^{2}\left(\Phi^{(q)} ; e^{-\frac{x}{r}}\right) & \text { if } q \leq 2 p, \\ O\left(n^{-2 p-1} \log n\right) E_{n-p-1}^{2}\left(\Phi^{(q)} ; e^{-\frac{x}{r}}\right) & \text { if } q=2 p+1, \\ O\left(n^{-2 p-1}\right) E_{n-p-1}^{2}\left(\Phi^{(q)} ; e^{-\frac{x}{r}}\right) & \text { if } q>2 p+1 .\end{cases}
$$

For $1 \leq x<\infty$ we use (2.7) with $k=0$ and $r>2$. We have

$$
\begin{aligned}
& \int_{1}^{\infty} e^{-\frac{x}{s}}\left[e^{-\frac{x}{r}}\left|f(x)-P_{n-1}(x)\right|\right]^{2} d x \\
& \quad \leq c\left[\int_{1}^{\infty} e^{-\frac{x}{s}} x^{2 p-q} d x\right] n^{-q} E_{n-p-1}^{2}\left(\Phi^{(q)} ; e^{-\frac{x}{r}}\right), \quad \frac{1}{s}+\frac{1}{r}=1 .
\end{aligned}
$$

Thus for $I_{1}$ the bounds (2.10), with $r>2$, hold.

To examine $I_{2}$, we apply to it the $n$-point Gauss-Laguerre formula,

$$
\int_{0}^{\infty} e^{-x} f(x) d x=\sum_{i=1}^{n} h_{n i} f\left(x_{n i}\right)+R_{n}^{\mathrm{GL}}(f),
$$

where the nodes $\left\{x_{n i}\right\}$ are in increasing order, i.e., $0<x_{n 1}<x_{n 2}<\cdots<$ $x_{n n}<\infty$. Since this latter integrates exactly any polynomial of degree $2 n-1$, recalling (2.7), we obtain, for $r>2$ and $s=r / 2$,

$$
\begin{aligned}
I_{2} & =\sum_{i=1}^{n} h_{n i}\left[f\left(x_{n i}\right)-P_{n-1}\left(x_{n i}\right)\right]^{2}=\sum_{i=1}^{n} h_{n i} e^{\frac{x_{n i}}{s}}\left[e^{-\frac{x_{n i}}{r}}\left|f\left(x_{n i}\right)-P_{n-1}\left(x_{n i}\right)\right|\right]^{2} \\
& \leq c\left(\sum_{i=1}^{n} h_{n i} e^{\frac{x_{n i}}{s}} x_{n i}^{2 p-q}\right) n^{-q} E_{n-p-1}^{2}\left(\Phi^{(q)} ; e^{-\frac{x}{r}}\right) .
\end{aligned}
$$

When $q \leq 2 p$, since (see [26])

$$
\lim _{n \rightarrow \infty} \sum_{i=1}^{n} h_{n i} e^{\frac{x_{n i}}{s}} x_{n i}^{2 p-q}=\int_{0}^{\infty} e^{-\left(1-\frac{2}{r}\right) x} x^{2 p-q} d x<\infty
$$


we have

$$
I_{2} \leq c n^{-q} E_{n-p-1}^{2}\left(\Phi^{(q)} ; e^{-\frac{x}{r}}\right) .
$$

When $q \geq 2 p+2$, to bound $I_{2}$, we first notice that

$$
I_{2} \leq c x_{n 1}^{2 p-q+2}\left(\sum_{i=1}^{n} h_{n i} e^{\frac{x_{n i}}{s}} x_{n i}^{-2}\right) n^{-q} E_{n-p-1}^{2}\left(\Phi^{(q)} ; e^{-\frac{x}{r}}\right) .
$$

Then, recalling (see $[24$, pp. 129,355$])$ that

$$
x_{n i} \sim \frac{(i+1)^{2}}{n+1}, \quad i=1, \ldots, n
$$

and

$$
h_{n i} \sim \frac{i}{n} \text { for } 0<x_{n i} \leq \omega,
$$

where $\omega$ is any fixed positive real constant, we note that

$$
\sum_{x_{n i} \leq \omega} h_{n i} \frac{e^{\frac{x_{n i}}{s}}}{x_{n i}^{2}} \leq c n
$$

and

$$
\sum_{x_{n i}>\omega} h_{n i} \frac{e^{\frac{x_{n i}}{s}}}{x_{n i}^{2}}<c \sum_{i=1}^{n} h_{n i} e^{\frac{x_{n i}}{s}} \leq c .
$$

Therefore, in this case we have

$$
I_{2} \leq c n^{-2 p-1} E_{n-p-1}^{2}\left(\Phi^{(q)} ; e^{-\frac{x}{r}}\right) .
$$

Similarly, when $q=2 p+1$, from the quantity

$$
\sum_{i=1}^{n} h_{n i} \frac{e^{\frac{x_{n i}}{s}}}{x_{n i}}
$$

we obtain the corresponding bound stated in (2.10).

Incidentally, we notice that when in (2.8) we take $q=p$, we get a bound which is similar to corresponding ones recently obtained in [4, 14].

The machinery we have used to prove Theorem 1 allows us to derive a new error estimate for the classical Gauss-Laguerre quadrature formula when this latter is applied to functions $f \in C_{p}^{q}[0, \infty), q \geq p \geq 0$. Indeed, by considering the same polynomial $P_{n-1}(x)$ defined in the proof of Theorem 1, we can write

$$
\left|R_{n}^{\mathrm{GL}}(f)\right| \leq \int_{0}^{\infty} e^{-x}\left|f(x)-P_{n-1}(x)\right| d x+\sum_{i=1}^{n} h_{n i}\left|f\left(x_{n i}\right)-P_{n-1}\left(x_{n i}\right)\right| .
$$

To examine the behavior of the two terms on the right-hand side, we proceed as we did for the quantities $I_{1}$ and $I_{2}$ in the proof of Theorem 1 . We obtain

Theorem 2. Let $f \in C_{p}^{q}[0, \infty), q \geq p \geq 0$. Then for the remainder term $R_{n}^{\mathrm{GL}}(f)$ of the Gauss-Laguerre formula we have the bounds

$$
\left|R_{n}^{\mathrm{GL}}(f)\right|= \begin{cases}O\left(n^{-\frac{q}{2}}\right) E_{n-p-1}\left(\Phi^{(q)} ; e^{-\frac{x}{2}}\right) & \text { if } q \leq 2 p+1, \\ O\left(n^{-p-1} \log n\right) E_{n-p-1}\left(\Phi^{(q)} ; e^{-\frac{x}{2}}\right) & \text { if } q=2 p+2 \\ O\left(n^{-p-1}\right) E_{n-p-1}\left(\Phi^{(q)} ; e^{-\frac{x}{2}}\right) & \text { if } q \geq 2 p+3\end{cases}
$$


Remark 1. If we examine the remainder term following the standard procedure, that is, we consider $f \in C^{p}[0, \infty)$ even when $q>p$ and take in (2.11) $P_{n-1}(x)$ coinciding for example with the polynomial defined in Lemma 2, after setting $x=t^{2}$ (see also [14, Theorem 1]), we would obtain the bound $O\left(n^{-\frac{p}{2}}\right) E_{n-p-1}^{*}\left(f^{(p)} ; e^{-t^{2}}\right)$.

Remark 2. The need of estimates of type (2.12) for functions $f \in C_{p}^{q}[0, \infty)$, with $q>p$, arises whenever $f(x)$ at the origin is not as smooth as elsewhere. For instance, in the case of an integral equation of type (1.1) with a kernel $K(x, y)=K_{s}(x-y)+K_{r}(x, y)$, where $K_{s}$ is weakly singular and $K_{r}$ is smooth, we would set

$$
\begin{aligned}
\int_{0}^{\infty} e^{-x} K(x, y) f(x) d x= & \int_{0}^{\infty} e^{-x} K_{s}(x-y) f(x) d x \\
& +\int_{0}^{\infty} e^{-x}\left[K_{r}(x, y) f(x)\right] d x
\end{aligned}
$$

The first integral on the right-hand side would then be approximated by an $n$ point product rule of type (2.13) below, and the last integral by the $n$-point Gauss-Laguerre formula.

Next we consider product rules of interpolatory type, based on the zeros of Laguerre polynomials, of the form

$$
\int_{0}^{\infty} e^{-x} K(x, y) f(x) d x=\sum_{i=1}^{n} w_{n i}(y) f\left(x_{n i}\right)+R_{n}^{K}(f ; y),
$$

where $R_{n}^{K}(f ; y)=\int_{0}^{\infty} e^{-x} K(x, y)\left[f(x)-L_{n}(f ; x)\right] d x$. By applying the Hölder inequality, we obtain

$$
\begin{aligned}
\int_{0}^{\infty} & e^{-x}|K(x, y)|\left|f(x)-L_{n}(f ; x)\right| d x \\
& \leq\left\{\int_{0}^{\infty} e^{-x}|K(x, y)|^{2} d x\right\}^{\frac{1}{2}}\left\{\int_{0}^{\infty} e^{-x}\left|f(x)-L_{n}(f ; x)\right|^{2} d x\right\}^{\frac{1}{2}},
\end{aligned}
$$

and, assuming

$$
\left\|e^{-\alpha y} \int_{0}^{\infty} e^{-x}|K(x, y)|^{2} d x\right\|_{\infty} \leq c \text { for some } \alpha \geq 0,
$$

from Theorem 1 we derive a uniform bound for $e^{-\frac{a}{2} y} R_{n}^{K}(f ; y)$.

Theorem 3. Let $f \in C_{p}^{q}[0, \infty), q \geq p \geq 0$. If in (2.13) the kernel $K(x, y)$ satisfies condition (2.14), then for any $r>2$ we have the bounds

$$
\begin{aligned}
\left\|e^{-\frac{\alpha}{2} y} R_{n}^{K}(f ; y)\right\|_{\infty} & = \begin{cases}O\left(n^{-\frac{q}{2}}\right) E_{n-p-1}\left(\Phi^{(q)} ; e^{-\frac{x}{r}}\right) & \text { if } q \leq 2 p, \\
O\left(n^{-p-\frac{1}{2}} \log n\right) E_{n-p-1}\left(\Phi^{(q)} ; e^{-\frac{x}{r}}\right) & \text { if } q=2 p+1, \\
O\left(n^{-p-\frac{1}{2}}\right) E_{n-p-1}\left(\Phi^{(q)} ; e^{-\frac{x}{r}}\right) & \text { if } q \geq 2 p+2 .\end{cases}
\end{aligned}
$$

Remark 3. Notice that in (2.15) we have the factor $n^{-p-\frac{1}{2}}$ while we would have expected the term $n^{-p-1}$ or $n^{-p-1+\varepsilon}$, with $\varepsilon>0$ as small as we like, as in (2.12). This is due to the fact that in our proof we have been forced to use the Hölder inequality with the $L^{2}$-norms.

Furthermore, for rule (2.13) the following property holds. 
Theorem 4. For any fixed $y \geq 0$ let $\int_{0}^{\infty} e^{-x}|K(x, y)|^{2} d x<\infty$. Furthermore, let $f \in C[0, \infty)$ be a function satisfying the inequality

$$
|f(x)| \leq c e^{(1-\varepsilon) \frac{x}{2}},
$$

for some $\varepsilon>0$. Then for any fixed $y \geq 0$ we have

$$
\lim _{n \rightarrow \infty} \sum_{i=1}^{n}\left|w_{n i}(y)\right| f\left(x_{n i}\right)=\int_{0}^{\infty} e^{-x}|K(x, y)| f(x) d x .
$$

Proof. By inspecting the proofs of Lemma 2 and relation (2.4) in [23], we discover that with very minor changes they also hold for our rule (2.13). Indeed, it is sufficient to consider in those proofs the $L^{2}$-norm $\|u\|_{2}=\left[\int_{0}^{\infty} e^{-x}|u(x)|^{2} d x\right]^{\frac{1}{2}}$, choose $h(x)=e^{(1-\varepsilon) \frac{x}{2}}$, use Lemma 3 , replace $k(x)$ by $e^{-x} K(x, y)$ and $k^{*}(x)$ by $e^{-x} K^{*}(x, y)$, and finally recall the sufficient condition obtained in [26] for convergence of the Gauss-Laguerre rule.

\section{NUMERICAL RESUlts}

In the first part of this section we apply the Gauss-Laguerre formula and the product rule (2.13) to some functions of class $C_{p}^{q}[0, \infty)$ to verify the order of our estimates (2.12) and (2.15).

In particular, to test our bounds (2.12), we have applied the $n$-point GaussLaguerre formula to the following integral:

$$
I_{1}=\int_{0}^{\infty} e^{-a x} x^{b} d x=\int_{0}^{\infty} e^{-x}\left[x^{b} e^{(1-a) x}\right] d x=\frac{\Gamma(b+1)}{a^{b+1}},
$$

with $b=\frac{1}{2}, \frac{3}{2}, \frac{5}{2}$ and $a=1, \frac{1}{2}$. The corresponding relative errors (in absolute values) are reported in Table 1 .

To check the behavior of formulas of type (2.13), we consider the integrals

$$
I_{2}=\int_{0}^{\infty} e^{-x} e^{-|x-y|} x^{b} d x
$$

and

$$
I_{3}=\int_{0}^{\infty} e^{-x} E_{1}(|x-y|) x^{b} d x,
$$

\begin{tabular}{|c|c|c|c|c|c|c|c|c|}
\hline$a$ & $b^{n}$ & 4 & 8 & 16 & 32 & 64 & 128 & 256 \\
\hline \multirow{3}{*}{1} & $1 / 2$ & $1.5 E-2$ & $5.2 E-3$ & $1.8 E-3$ & $6.4 E-4$ & $2.3 E-4$ & $8.0 E-5$ & $2.8 E-5$ \\
\hline & $3 / 2$ & $2.4 E-3$ & $3.8 E-4$ & $6.3 E-5$ & $1.1 E-5$ & $1.9 E-6$ & $3.4 E-7$ & $5.9 E-8$ \\
\hline & $5 / 2$ & $6.5 E-4$ & $4.3 E-5$ & $3.3 E-6$ & $2.8 E-7$ & $2.4 E-8$ & $2.1 E-9$ & $1.8 E-10$ \\
\hline \multirow{3}{*}{0.5} & $1 / 2$ & $3.2 E-3$ & $1.7 E-3$ & $6.3 E-4$ & $2.2 E-4$ & $8.0 E-5$ & $2.8 E-5$ & $1.0 E-5$ \\
\hline & $3 / 2$ & $1.1 E-2$ & $6.4 E-5$ & $1.0 E-5$ & $1.9 E-6$ & $3.3 E-7$ & $5.9 E-8$ & $1.0 E-8$ \\
\hline & $5 / 2$ & $4.4 E-2$ & $4.6 E-5$ & $2.6 E-7$ & $2.3 E-8$ & $2.0 E-9$ & $1.8 E-10$ & $1.6 E-11$ \\
\hline
\end{tabular}

where $E_{1}(t)$ denotes the exponential integral (see [1]). Their kernels satisfy condition (2.14) with $\alpha=0$ and appear in certain well-known Wiener-Hopf integral equations (see $[2,13,22]$ ).

TABLE 1. Relative errors of the $n$-point Gauss-Laguerre rule 
We recall (see [10]) that the coefficients $w_{n i}(y)$ of $(2.13)$ can be represented as follows:

$$
w_{n i}(y)=h_{n i} \sum_{j=0}^{n-1} a_{j}(y) L_{j}\left(x_{n i}\right), \quad a_{j}(y)=\int_{0}^{\infty} e^{-x} K(x, y) L_{j}(x) d x .
$$

Furthermore, in the case of our kernels the following properties of Laguerre polynomials, which are taken from $[24, \S 5.1]$, are fundamental:

$$
\left\{\begin{array}{l}
L_{0}(x)=1, \\
L_{1}(x)=1-x, \\
j L_{j}(x)=(2 j-1-x) L_{j-1}(x)-(j-1) L_{j-2}(x), \quad j=2, \ldots,
\end{array}\right.
$$

$$
\begin{gathered}
L_{j}(0)=1, \\
L_{j}(x)=L_{j}^{(1)}(x)-L_{j-1}^{(1)}(x), \\
\frac{d}{d x} L_{j}(x)=-L_{j-1}^{(1)}(x), \\
j\left[L_{j}(x)-L_{j-1}(x)\right]=-x L_{j-1}^{(1)}(x),
\end{gathered}
$$

where $L_{j}^{(1)}(x)$ denotes the generalized Laguerre polynomial orthogonal with respect to the weight function $x e^{-x}$. Notice that from (3.7) and (3.8) we obtain

$$
\frac{d}{d x}\left(e^{-x} L_{j}(x)\right)=-e^{-x} L_{j}^{(1)}(x) .
$$

Then we introduce the auxiliary quantities

$$
\mu_{j}(y)=\int_{0}^{\infty} e^{-x} K(x, y) L_{j}^{(1)}(x) d x=-\int_{0}^{\infty} K(x, y) d\left(e^{-x} L_{j}(x)\right),
$$

so that, by (3.7), for $j \geq 1$ we have

$$
a_{j}(y)=\mu_{j}(y)-\mu_{j-1}(y) .
$$

We consider first the case $K(x, y)=e^{-|x-y|}$. For $y \geq 0$, by applying the integration-by-parts rule, we obtain

$$
\begin{aligned}
\mu_{j}(y) & =\mu_{j}^{(2)}(y)=-\int_{0}^{y} e^{-(y-x)} d\left(e^{-x} L_{j}(x)\right)-\int_{y}^{\infty} e^{-(x-y)} d\left(e^{-x} L_{j}(x)\right) \\
& =e^{-y}\left[1+L_{j}(y)-L_{j+1}(y)-D_{j}(y)\right],
\end{aligned}
$$

where the quantities

$$
D_{j}(y)=e^{2 y} \int_{y}^{\infty} e^{-2 x} L_{j}(x) d x
$$

satisfy the recurrence relation

$$
\left\{\begin{array}{l}
D_{0}(y)=\frac{1}{2} \\
D_{j}(y)=\frac{1}{2}\left[D_{j-1}(y)+L_{j}(y)-L_{j-1}(y)\right], \quad j=1,2, \ldots
\end{array}\right.
$$


TABLE 2. Relative errors of (2.13) applied to $I_{2}$

\begin{tabular}{|c|c|cccccl|}
\hline$y$ & $b^{n}$ & 4 & 8 & 16 & 32 & 64 & \multicolumn{1}{c|}{128} \\
\hline \multirow{3}{*}{$1.0 E-2$} & $1 / 2$ & $5.2 E-2$ & $1.6 E-2$ & $5.4 E-3$ & $1.8 E-3$ & $5.9 E-4$ & $1.8 E-4$ \\
& $3 / 2$ & $2.7 E-2$ & $2.9 E-3$ & $4.1 E-4$ & $6.4 E-5$ & $1.1 E-5$ & $1.7 E-6$ \\
& $7 / 2$ & $2.6 E-1$ & $5.6 E-4$ & $8.2 E-6$ & $2.3 E-7$ & $7.9 E-9$ & $2.7 E-10$ \\
\hline & $1 / 2$ & $1.5 E-3$ & $2.8 E-3$ & $1.5 E-3$ & $3.9 E-4$ & $1.4 E-4$ & $4.2 E-5$ \\
1 & $3 / 2$ & $9.3 E-3$ & $1.6 E-4$ & $1.2 E-4$ & $5.9 E-6$ & $1.4 E-6$ & $2.6 E-7$ \\
& $7 / 2$ & $2.8 E-1$ & $3.4 E-5$ & $1.2 E-5$ & $1.6 E-7$ & $4.0 E-9$ & $7.3 E-11$ \\
\hline \multirow{3}{*}{10} & $1 / 2$ & $7.1 E-3$ & $4.6 E-3$ & $1.6 E-4$ & $1.2 E-4$ & $2.2 E-5$ & $3.4 E-6$ \\
& $3 / 2$ & $1.8 E-3$ & $4.7 E-4$ & $4.6 E-6$ & $3.0 E-6$ & $1.6 E-7$ & $8.3 E-9$ \\
& $7 / 2$ & $3.7 E-3$ & $4.1 E-5$ & $6.0 E-8$ & $8.4 E-9$ & $8.9 E-11$ & $8.7 E-13$ \\
\hline
\end{tabular}

Therefore, from (3.12) we have the expressions

$$
\left\{\begin{aligned}
a_{0}(y)=a_{0}^{(2)}(y) & =e^{-y}\left(y+\frac{1}{2}\right), \\
a_{j}(y)=a_{j}^{(2)}(y)=e^{-y}\left[-L_{j+1}(y)+2 L_{j}(y)-L_{j-1}(y)-D_{j}(y)+D_{j-1}(y)\right], & j=1,2, \ldots,
\end{aligned}\right.
$$

which are needed to construct the coefficients $w_{n i}(y)$ (see (3.4)) of the corresponding product rule (2.13).

In Table 2 we report the estimated relative errors ${ }^{3}$ at the points $y=10^{-2}, 1$, 10 , for $b=\frac{1}{2}, \frac{3}{2}, \frac{7}{2}$ and $n=2^{k}, k=2, \ldots, 7$.

Next we construct the coefficients $w_{n i}(y)$ of $(2.13)$ when this latter is applied to the integral $I_{3}$ in (3.3). To derive an expression for the quantities $a_{j}(y)=$ $a_{j}^{(3)}(y)$ in (3.4), we examine first

$$
\begin{aligned}
\mu_{j}^{(3)}(y) & =\int_{0}^{\infty} e^{-x} E_{1}(|x-y|) L_{j}^{(1)}(x) d x \\
& =-\int_{0}^{y} E_{1}(y-x) d\left(e^{-x} L_{j}(x)\right)-\int_{y}^{\infty} E_{1}(x-y) d\left(e^{-x} L_{j}(x)\right)
\end{aligned}
$$

when $y>0$.

Since (see [1]) $\frac{d}{d v} E_{1}(v)=-\frac{e^{-v}}{v}$, by applying the integration-by-parts rule, we obtain

$$
\mu_{j}^{(3)}(y)=E_{1}(y)-Q_{j}(y), \quad j=0,1, \ldots
$$

where the quantity

$$
Q_{j}(y)=f_{0}^{\infty} e^{-x} \frac{e^{-|x-y|}}{x-y} L_{j}(x) d x
$$

satisfies the recurrence relation

$$
\left\{\begin{array}{l}
Q_{0}(y)=-e^{-y}(\gamma+\log 2 y), \\
Q_{1}(y)=(1-y) Q_{0}(y)-a_{0}^{(2)}(y), \\
(j+1) Q_{j+1}(y)=(2 j+1-y) Q_{j}(y)-j Q_{j-1}(y)-a_{j}^{(2)}(y), \quad j=1,2, \ldots .
\end{array}\right.
$$

${ }^{3}$ These are obtained by taking as exact value the approximation given by the same rule with $n=360$ nodes. 
TABLE 3. Relative errors of (2.13) applied to $I_{3}$

\begin{tabular}{|c|c|cccccc|}
\hline$y$ & $n$ & 4 & 8 & 16 & 32 & 64 & 128 \\
\hline \multirow{3}{*}{$1.0 E-2$} & $1 / 2$ & $2.0 E-1$ & $8.4 E-2$ & $3.3 E-2$ & $1.2 E-2$ & $4.0 E-3$ & $9.7 E-4$ \\
& $3 / 2$ & $2.0 E-1$ & $3.4 E-2$ & $6.1 E-3$ & $1.1 E-3$ & $1.7 E-4$ & $1.6 E-5$ \\
& $7 / 2$ & $5.3 E-0$ & $3.2 E-2$ & $8.9 E-4$ & $3.1 E-5$ & $9.8 E-7$ & $7.0 E-9$ \\
\hline & $1 / 2$ & $1.9 E-2$ & $1.4 E-3$ & $1.6 E-3$ & $1.2 E-5$ & $4.9 E-6$ & $4.9 E-7$ \\
1 & $3 / 2$ & $2.3 E-2$ & $2.5 E-4$ & $2.7 E-4$ & $1.9 E-5$ & $3.3 E-6$ & $4.2 E-7$ \\
& $7 / 2$ & $9.3 E-1$ & $5.8 E-4$ & $7.6 E-5$ & $1.7 E-6$ & $8.1 E-8$ & $2.7 E-9$ \\
\hline & $1 / 2$ & $7.5 E-3$ & $1.1 E-2$ & $1.6 E-3$ & $8.4 E-4$ & $1.4 E-4$ & $2.4 E-6$ \\
10 & $3 / 2$ & $1.6 E-3$ & $8.4 E-4$ & $5.3 E-5$ & $1.3 E-5$ & $1.1 E-6$ & $1.8 E-8$ \\
& $7 / 2$ & $4.0 E-3$ & $5.4 E-5$ & $5.2 E-7$ & $2.7 E-8$ & $5.0 E-10$ & $2.5 E-12$ \\
\hline
\end{tabular}

The sign $f$ in (3.16) means that the integral is defined in the Cauchy principal value sense. The letter $\gamma$ in (3.17) denotes the Euler-Mascheroni constant $0.57721566490153286 \ldots$. Thus, from (3.12) we have

$$
\left\{\begin{array}{l}
a_{0}^{(3)}(y)=E_{1}(y)-Q_{0}(y), \\
a_{j}^{(3)}(y)=-Q_{j}(y)+Q_{j-1}(y), \quad j=1,2, \ldots .
\end{array}\right.
$$

When $y=0$ we need to consider the quantity

$j\left[\mu_{j}^{(3)}(0)-\mu_{j-1}^{(3)}(0)\right]=-\int_{0}^{\infty} E_{1}(x) d\left(e^{-x} j\left[L_{j}(x)-L_{j-1}(x)\right]\right), \quad j=1,2, \ldots$,

and recall relation (3.9); we obtain

$$
j\left[\mu_{j}^{(3)}(0)-\mu_{j-1}^{(3)}(0)\right]=\int_{0}^{\infty} E_{1}(x) d\left(e^{-x} x L_{j-1}^{(1)}(x)\right)=\int_{0}^{\infty} e^{-x}\left[e^{-x} L_{j-1}^{(1)}(x)\right] d x .
$$

By rewriting the last integral in the form (see (3.10))

$$
-\int_{0}^{\infty} e^{-x} d\left(e^{-x} L_{j-1}(x)\right),
$$

and applying to it the integration-by-parts rule, we finally have

$$
j\left[\mu_{j}^{(3)}(0)-\mu_{j-1}^{(3)}(0)\right]=1-D_{j-1}(0),
$$

that is,

$$
a_{j}^{(3)}(0)=\left(1-2^{-j}\right) / j, \quad j=1,2, \ldots
$$

For the coefficient $a_{0}^{(3)}(0)$ we obtain the value

$$
a_{0}^{(3)}(0)=\log 2 .
$$

In Table 3 we report the estimated relative errors at the point $y=10^{-2}, 1$, 10 , for $b=\frac{1}{2}, \frac{3}{2}, \frac{7}{2}$ and $n=2^{k}, k=2, \ldots, 7$.

According to Remark 3, when we apply our product rule to integrals $I_{2}$ and $I_{3}$ defined in (3.2) and (3.3), we should expect a convergence rate of type $O\left(n^{1+b}\right)$, or $O\left(n^{1+b-\varepsilon}\right)$ with $\varepsilon>0$ as small as we like. The behavior of the errors reported in Tables 2 and 3 seems to confirm this estimate. 
TABLE 4. Relative errors for $u(0)$

\begin{tabular}{|c|ccccc|}
\hline$n$ & 4 & 8 & 16 & 32 & 64 \\
\hline rel.err. & $3.6 E-3$ & $1.3 E-3$ & $4.0 E-4$ & $1.2 E-4$ & $3.3 E-5$ \\
\hline
\end{tabular}

Finally, we consider one further application of the product rule used in the last example. We use it to construct a Nyström interpolant for the following case of the well-known linear transport equation (see $[2,13])$ :

$$
u(y)-\frac{1}{4} \int_{0}^{\infty} E_{1}(|x-y|) u(x) d x=\frac{1}{2},
$$

whose solution at $x=0$ assumes the known value $u(0)=\frac{\sqrt{2}}{2}$.

Since it is known (see [13]) that $\lim _{x \rightarrow \infty} u(x)=1$ and $u(x)-1=o\left(e^{-\mu x}\right)$ as $x \rightarrow \infty$, for any $0<\mu<\mu^{*}, \mu^{*}=0.957504$, we set $\bar{u}(x)=u(x)-1$ and rewrite (3.21) in the following form:

$$
\bar{u}(y)-\frac{1}{4} \int_{0}^{\infty} e^{-x} E_{1}(|x-y|)\left[e^{x} \bar{u}(x)\right] d x=-\frac{1}{4}\left[e^{-y}-y E_{1}(y)\right] .
$$

We recall that $\bar{u} \in C_{0}^{\infty}[0, \infty)$ and, furthermore, as $x \rightarrow 0$ the solution $\bar{u}(x)$ behaves like $c+x^{b}$ for any $0<b<1$ (see [13]). (Actually, it appears (see [2]) that $\vec{u}(x) \sim c+x \log x$.)

In Table 4 we report the relative accuracy we have obtained by our Nyström interpolant at the origin for some values of $n$.

We have also evaluated the interpolant at $x=1,2, \ldots, 10$ and noticed that as we move away from the point $x=0$ the accuracy seems to improve slightly, as predicted by the values reported in Table 3 .

All computations have been performed on a personal computer, using 16-digit arithmetic.

\section{BIBLIOGRAPHY}

1. M. Abramowitz and I. A. Stegun, Handbook of mathematical functions, NBS Appl. Math. Ser., no. 55, Washington, DC, 1964.

2. I. K. Abu-Shumays and E. H. Bareiss, Adjoining appropriate singular elements to transport theory computations, J. Math. Anal. Appl. 48 (1974), 200-222.

3. J. Balázs and P. Turán, Notes on interpolation. VIII, Acta Math. Acad. Sci. Hungar. 12 (1961), 469-474.

4. K. Balázs and I. Joó, On speed of mean convergence of Lagrange and Hermite interpolation based on the roots of Laguerre polynomials, Publ. Math. Debrecen 38 (1991), 247-253.

5. K. Balázs and T. Kilgore, Pointwise exponentially weighted estimates for approximation on the half-line, Numer. Funct. Anal. Optim. 13 (1992), 223-232.

6. G. Criscuolo, G. Mastroianni, and G. Monegato, Convergence properties of a class of product formulas for weakly singular integral equations, Math. Comp. 55 (1990), 213-230.

7. R. Dahlhaus, Pointwise approximation by algebraic polynomials, J. Approx. Theory 57 (1989), 274-277.

8. P. J. Davis and P. Rabinowitz, Methods of numerical integration, 2nd ed., Academic Press, New York, 1984.

9. Z. Ditzian and V. Totik, Moduli of smoothness, Springer Ser. Comput. Math., vol. 9, Springer, Berlin, 1987. 
10. D. Elliott and D. F. Paget, Product integration rules and their convergence, BIT 16 (1976), 32-40.

11. G. Freud, Orthogonal polynomials, Pergamon Press, Oxford, 1971.

12. I. Gopengauz, $A$ theorem of $A$. F. Timan on the approximation of functions by polynomials on a finite segment, Math. Notes 1 (1967), 110-116.

13. I. G. Graham and W. R. Mendes, Nyström-product integration for Wiener-Hopf equations with applications to radiative transfer, IMA J. Numer. Anal. 9 (1989), 261-284.

14. I. Joó and N. X. Ky, Answer to a problem of Paul Turán, Ann. Univ. Sci. Budapest. Sect. Math. 31 (1991), 229-241.

15. A. Knopfmacher and D. S. Lubinsky, Mean convergence of Lagrange interpolation for Freud's weights with application to product integration rules, J. Comput. Appl. Math. 17 (1987), 79-103.

16. D. S. Lubinski and A. Sidi, Convergence of product integration rules for functions with interior and endpoint singularities over bounded and unbounded intervals, Math. Comp. 46 (1986), 229-245.

17. D. S. Lubinsky, Convergence of product integration rules for weights on the whole real line, Contributions in Numerical Mathematics, Vol. 2, World Scientific, Singapore, 1993, pp. 255-270.

18. Y. L. Luke, Mathematical functions and their approximations, Academic Press, New York, 1975.

19. G. Mastroianni and G. Monegato, Polynomial approximations of functions with endpoint singularities and product integration formulas, Math. Comp. 62 (1994), 725-738.

20. G. Monegato and V. Colombo, Product integration for the linear transport equation in slab geometry, Numer. Math. 52 (1988), 219-240.

21. P. Nevai, Mean convergence of Lagrange interpolation. I, J. Approx. Theory 18 (1976), 363-377.

22. I. H. Sloan and A. Spence, Projection methods for integral equations on the half-line, IMA J. Numer. Anal. 6 (1986), 153-172.

23. W. E. Smith, I. H. Sloan, and A. H. Opie, Product integration rules over infinite intervals. I, Rules based on the zeros of Hermite polynomials, Math. Comp. 40 (1983), 519-535.

24. G. Szegö, Orthogonal polynomials, Amer. Math. Soc. Colloq. Publ., vol. 23, Amer. Math. Soc., Providence, RI, 1975.

25. A. F. Timan, Theory of approximation of functions of a real variable, Pergamon Press, New York and Macmillan, New York, 1963.

26. J. V. Uspenksy, On the convergence of quadrature formulas related to an infinite interval, Trans. Amer. Math. Soc. 30 (1928), 542-559.

27. G. Vainikko and A. Pedas, The properties of solutions of weakly singular integral equations, J. Austral. Math. Soc. Ser. B 22 (1980/81), 419-430.

Dipartimento di Matematica, Universita della Basilicata, I-85100 Potenza, Italy

E-mail address: Mastroianni@pzvx85.cineca.it

Dipartimento di Matematica, Politecnico di Torino, I-10129 Torino, Italy

E-mail address: Monegato@itopoli.bitnet 